\title{
Gardosian patterns in tribology
}

\author{
Dr. Christopher DellaCorte \\ National Aeronautics and Space Administration, Glenn Research Center, Cleveland, OH 44135, USA
}

The following paper is a memorial retrospective on selected research of Dr. Michael N. Gardos. Dr. Gardos spent his professional career engaged in tribological research which often extended the scientific boundaries of the field. Several of the concepts he put forth into the tribology community were initially met with grave skepticism but over time his views have been largely embraced but not widely acknowledged. His approach to new research topics was often characterized by these qualities: 1) pioneering points of view, 2) the use of the model experiment, and 3) the presence of multiple research agendas for each single experiment.

I have chosen to name his research approach as "Gardosian Patterns" in honor of his contributions to Tribology. Three specific examples of these patterns will be reviewed. One is the concept of atomic level tailoring of materials to control macroscopic properties. A second is the use of a model ball polishing experiment to identify high fracture toughness ceramics for use in rolling element bearings. A third Gardosian Pattern example is his pioneering work with the tribology of diamond and diamond films in which he proposed controlling friction via surface bond tailoring. In these examples, Gardos utilized conventional research tools in unconventional ways and, at times, even developed new tools which have become part of the mainstream. His remarkable career has left a positive and lasting mark on Tribology.

KEY WORDS: tribology, friction, wear, ball bearing, ceramics, diamond, modeling

\section{Introduction}

Tribology is a broad and complex field which often presents vexing challenges to research scientists. Rarely does one encounter a tribological problem which can be classified as purely mechanical or chemical or electrical. More likely, combinations of phenomena are responsible for the tribological response observed in most systems. For instance consider the tribology of a piston ring sliding against an oil-lubricated cylinder wall an internal combustion engine. Mechanical design of the ring profile, gas pressures and linear velocity play a critical role in the tribological success or failure of this contact. So too however, does the additive chemistry of the oil and interactions with the combustion environment $[1,2]$. The proper 
engineering of such a tribological contact necessitates an interdisciplinary approach. The tribologist must be cognizant of many facets of science and engineering. Dr. M.N. Gardos epitomized the interdisciplinary nature of the field.

\section{Education and professional history}

Dr. Gardos received his primary and secondary education in Hungary and immigrated to the United States to join his father in 1959. After a brief stay in California, Gardos joined the US Army and worked as a fuel and lubricants test technician stationed in Germany. It was during this period that he was first introduced to the field that would occupy the remainder of his career. Following his military service, Gardos studied at the University of California under the GI bill and upon graduation went to work for Hughes Aircraft Company as a staff researcher.

Over the course of the next thirty years, Gardos worked on numerous research programs related to aerospace systems. This research can be tracked by examining his publications which covered topics broadly ranging from solid film lubricant coatings and greases for space mechanisms on satellites to corrosion and erosion of missile radomes. His publications are characterized by simultaneously including practical engineering approaches to solving tribology problems as well as scientific studies capable of extending the knowledge base. Gardos routinely immersed himself in the scientific literature before embarking on a new research topic and had the rare ability to instantly recall important citations and journals on numerous subjects. He truly espoused the notion that to move forward one has to first learn what others on the same path had already learned. Many of us today do not go to such great lengths to read the pertinent literature and find ourselves reinventing square wheels.

Upon careful reflection, I have decided to concentrate my review on three specific tribology subjects which dominated Gardos' later career. One relates to a theory he put forth that one could tailor the macroscopic shear properties of a solid, in this case rutile, through the atomic level manipulations of lattice vacancies in order to transform an abrasive material into a lubricant [3]. Certainly this concept of atomic level features controlling macroscopic properties was not novel but Gardos' application to solid lubrication and his a priori theoretical model followed by experiment helped set the tribology stage for many atomistic modeling efforts now underway.

A second subject, not widely recognized, centered on an engineering approach to identify promising silicon nitride ceramics for rolling element bearings. At the time of this research the use of ceramics for ball bearings was not yet an 
accepted practice. Sporadic and unpredictable performance made their future commercialization uncertain. Gardos used this uncertainty to undertake innovative polishing experimental data for a wide range of ceramics and couple this data with fracture toughness information to identify probable candidate materials for ball bearings slated for extreme condition applications [4]. By cleverly selecting specimen sizes, the ceramic test specimen balls which resulted were compatible with key bearing test facilities. This greatly assisted the transition of ceramic hybrid bearings into a very high profile cryogenic bearing application.

The third subject upon which I will consider is the body of Gardos' work which centered on the study of how surface bonding, particularly in diamond and other carbon materials, affects friction [5]. Again, his work in this area wasn't the first but explored much of what is currently deemed Diamond-Like-Carbon tribology. Further, his diamond work included both thought experiments and laboratory experiments which helped bolster his theories. It is my intent through this paper to help the tribology community appreciate the positive legacy Gardos had on the field, offer appropriate credit for his work and to professionally acknowledge his contributions.

\section{Atomic level tailoring of friction}

In 1989, Gardos published an in depth literature review concerning titanium dioxide $\left(\mathrm{TiO}_{2}\right)$ which, hereafter, I will generally refer to as rutile in which he put forth the concept of atomic level tailoring to control shear and friction properties.(3). This concept was the culmination of many years worth of effort by him and others to develop high temperature solid lubricants based upon low shear strength glazes and oxides [6,7]. In this review, Gardos suggested that an experimentally driven "Edisonian" approach to lubricant development was certain to fail because many complex factors such

as ambient temperature, humidity, counterface chemistry and shear rates can affect shear behavior. In essence, without a scientifically based understanding of what controls oxide shear and a clear definition of the anticipated tribological environment, it is unlikely that a successful lubricious oxide lubricant will be found.

Rather, Gardos proposed a predictive model for the shear behavior of $\mathrm{TiO}_{2}$ in which its shear strength would be controlled by anion vacancies. These vacancies would then be controlled by environmental means (temperature and atmosphere) to influence the tribological properties. Based purely upon literature data, Gardos proposed a series of hypothetical lubricious oxides all of which were rutile at varying levels of oxygen deficiencies $\left(\mathrm{TiO}_{2}-\mathrm{x}\right)$. In his paper, he 
subtly pointed out that rutile was an excellent material for study and atomic manipulation because of its many, well studied and defined crystallographic forms. Whether rutile could be transformed from an abrasive pigment to a true and useful solid lubricant was in question.

To ascertain rutile's tribological properties, Gardos developed a unique test rig; a pin-on-plate tribometer located inside a Knudsen cell-like chamber which itself was placed inside a Scanning Electron microscope [8]. To this author's knowledge, at that time, no such combination of test capabilities had ever been undertaken. Individually, each component, the tribometer, the differentially pumped Knudsen cell and the SEM, were well developed but merging them into the same device was novel and challenging. This unique facility offered Gardos and his co-workers unprecedented latitude in controlling the experiment and examining the results in real time. Figure 1 shows the schematic details of the high temperature SEM tribometer.

The use of this novel testing approach and apparatus allowed Gardos to ascertain the tribo-performance of rutile. More importantly, the rig was used to alter the atomic structure during the test via temperature and atmospheric conditions and measure the effects on tribological behavior. Figure 2 shows just such a result. In this case, heating during sliding in both vacuum and air resulted in changes in shear strength (friction) predicted in Gardos' earlier paper. This is an impressive result. The test conditions of high temperature coupled with high vacuum reduced the oxygen content of the rutile and this had the anticipated effect on friction. As corroboration for the oxygen vacancies created during the test, Gardos observed that the optical properties of the specimens did indeed change indicating stoichiometric alterations had occurred.

This oxide tribology work demonstrated that one could indeed alter atomic level structure of a material and effect changes to its tribological behavior. This work opened the tribology community's eyes to the concept of atomic manipulation, work that continues to this day. Further, some researchers looked for ways to preserve the low friction structure of rutile under a wider range of test conditions thereby creating a tailored oxide solid lubricant. This work has met with some success but it has not been universal [9]. Nonetheless, Gardos' pioneering experiments show a path which may bear fruit for many years to come.

\section{Silicon-nitride ball polishing experiments leads to improved SSME bearings}

In the early days of NASA's Space Shuttle a persistent tribological challenge was excessive wear of the cryogenic turbopumps which feed fuel and oxidizer to the main engines. These pumps operate at high speed and utilize the liquid 
oxygen (LOX) or liquid hydrogen $\left(\mathrm{LH}_{2}\right)$ as their coolant and lubricant. The use of oils and greases is precluded because of safety, temperature and compatibility issues. The design approach consisted of 440C stainless steel ball bearings with PTFEglass composite cages. In use, the cages were expected to wear and deposit solid lubricants to the balls and then from the balls to the races [10]. In practice, the solid lubrication appeared to be marginal. Ball wear, especially for the LOX pumps, was high leading to undesirable geometric changes in the bearing. This led to increased heat generation and oxidation of the ball surface indicating severe ball surface heating. One concern considered at the time was the possibility of ignition of the ball material in the oxygen environment. Although no bearing failures were observed, bearing replacements were being done after only one or two flights rather than the initial design goal of at least seven flights.

Numerous solutions were proposed to address this problem. These included pressurizing the bearing cavity further and increasing the cryogen flow rate to reduce the occurrence of gaseous oxygen, improved solid lubrication, changing the ball material to more wear resistant metals and replacing the ball bearings with fluid film or hydrostatic bearings. A bearing test rig was used at NASA's Marshall Space Flight Center to evaluate these and other concepts. Another approach was forwarded to replace the all metal bearings with ceramic hybrid bearings utilizing silicon nitride rolling elements. This approach was considered radical as hybrid bearings, especially in man rated and/or cryogenic applications was yet unproven technology.

Gardos firmly believed ceramic hybrid bearings would alleviate the Space Shuttle Main Engine (SSME) turbopump bearing problem. Many in the technical community did not share his view citing concerns regarding silicon nitride's brittle behavior and the inability to manufacture high grade bearing balls. There was even a reluctance to test such a bearing over concerns that a catastrophic failure of the rolling elements could irreparably damage the test facility. Rather than directly address the criticism surrounding the use of ceramic hybrid ball bearings for the SSME, Gardos took a different, more subtle approach.

Gardos proposed and won an US Department of Defense funded contract to study the relationship between silicon nitride's fracture toughness (and hardness) to its wear resistance [11]. He cleverly designed an experiment based upon ceramic ball finishing (polishing) to gather polishing wear rate data and used the then state-of-the-art indentation hardness tests to measure hardness and to estimate fracture toughness. In his work, Gardos evaluated seven of the best silicon nitride ceramics then available. The results showed a remarkable correlation between a well accepted wear model, based upon the toughness and hardness data he measured, and the observed polishing wear data. Figure 3, adapted from reference 4 shows this data. 
More remarkable than the results, however, were Gardos' secondary agenda for the data and the test specimens. The ball polishing experiments resulted in several complete sets of silicon nitride bearing balls in the exact size and grade to fit NASA's LOX bearing test facility. At the time of this work, the there were general concerns amongst the technical community that ceramic bearings would suffer from brittle fracture induced failure and that ball surface finish requirements needed for critical applications such as cryogenics could not be met. By conducting these pioneering ceramic ball polishing experiments, Gardos and his colleagues were able to identify several ceramics with good fracture toughness and displayed the ability to be polished to surface finishes acceptable for critical ball bearing applications. Further, his data clearly showed one type of silicon nitride from one Japanese source to be the most wear resistant. The bearing balls were then offered to NASA for experimental testing. When evaluated along with other steels and ceramics, the material identified in Gardos' experiments performed well lasting fourteen times longer than the baseline $440 \mathrm{C}$ bearing balls and fully twice the then leading silicon nitride candidate selected by NASA. Currently, the SSME pumps use ceramic hybrid bearings with balls made from the Japanese material Gardos identified over a decade before.

Certainly, many researchers contributed to the successful implementation of ceramic hybrid bearings in the SSME turbopumps. Perhaps, the selected ball material would have been identified without Gardos' early experiments. This author, however, is a primary reference source during this period and well remembers the important and largely unknown role Gardos selflessly played in solving this national tribological problem. The innovative use of an engineering process like ball polishing to link basic materials science to an end application is a hallmark of Gardos' research style. One we can all learn from.

\section{Carbon-diamond-hydrogen tribology}

A third example of Gardos' pioneering efforts in tribology deals with his fascination and study of carbon, especially diamond, as a tribological material. Long used for miniature bearing surfaces and macroscopic metalworking tools, diamond and other hard carbon forms of this material have been the subject of much tribological research. In many applications which require the seemingly disparate combination of hardness, high thermal conductivity, high electrical resistivity and high optical transmittance properties diamond is a leading candidate. Over the course of his career, Gardos dealt with his share of 
these applications and thus had numerous opportunities to conduct research with diamond and diamond-like carbon. In his work, Gardos often looked beyond experimental data seeking fundamental bases for diamond's tribological behavior.

By carefully reviewing the work of others, he began to see a rather unique picture of this complex material. He often regaled eager audiences, including the author, with his view of diamond as a hard, thermally conductive bearing surface which actively formed adsorbed surface films of water and other hydrogen rich species which acted to reduce friction [5]. In fact, colleagues goodheartedly ridiculed Gardos' pantomime-like rendition of "hungry dangling bonds" on "unsatisfied" diamond surfaces long before diamond and diamond-like carbons coatings became household terms.

Further, Gardos combined his insatiable appetite for reading the diamond literature with his unique approach to in-situ SEM tribometry. His model experiments, in which sliding occurred at varying temperatures in vacuum and hydrogen, helped to elucidate the mechanisms which give rise to the complex tribological response of diamond. Figure 4 taken from reference 5, for instance, shows how the friction coefficient in hydrogen rises as the temperature changes the nature of the surface species present during sliding. In this case, low friction is again observed when the temperature drops allowing re-adsorption of hydrogen to occur forming tribologically beneficial hydrogen-carbon bonds at the surface. In his work, Gardos built upon the careful surface science studies of others, like Pepper [12], who had shown earlier that exposing diamond to high temperature vacuum and hydrogen transforms its electronic surface structure and surface bonding affecting friction.

These types of insightful experiments were only possible through the use of innovative tribology experiments in which many factors, normally held constant such as atmospheric pressure and chemistry, are purposefully altered. To do this judiciously required first an understanding of the tribological-material system and some hypothesis to test. For diamond and carbon tribology, Gardos' models and experiments added significantly to our understanding of this important class of materials.

\section{Concluding observations}

Dr. Michael N. Gardos made significant contributions to the field of tribology in many regards. He was an excellent experimentalist who often looked for meaning beyond the simple technical data which experiments often yield. He held the work and knowledge of others is the highest regard and read the scientific literature voraciously so that he could "stand on their shoulders" to move his own understanding forward. He was deeply dedicated to sharing his understanding and thought 
processes with others, at times when they were welcome or not. He demanded technical excellence in himself and from others. Gardos never settled for almost knowing an answer. If questions remained he doggedly pursued other means to arrive at a conclusion in a way that is captured by the phrase "Gardosian Patterns". He left his mark on Tribology and positively impacted many lives. His work lives on in the literature and his memory lives on in the hearts and minds of many.

\section{References}

[1] K.C. Radil, "The Influence of Honing on the Wear of Ceramic Coated Piston Rings and Cylinder Liners,” NASA/TM-2000-209794, February 2000, Lubrication Engineering, vol. 57, no. 7, pp. 10-12, July 2001.

[2] K.C. Radil, “Test Method to Evaluate Cylinder Liner-Piston Ring Coatings for Advanced Heat Engines,” NASA TM-107526 and ARLMR-362, May 1996.

[3] M.N. Gardos, "The Effect of Anion Vacancies on the Tribological Properties of Rutile (TiO2-x)," STLE Tribology Transactions, vol. 31, 4, pp. 427436, 1988 .

[4] M.N. Gardos, and R.G. Hardisty, "Fracture Toughness and Hardness Dependent Polishing Wear of Silicon Nitride Ceramics," Tribology Transactions, vol. 36, no. 4, pp. 653-660, October 1993.

[5] M.N. Gardos, "Re(de)construction-induced friction signatures of polished polycrystalline diamond films in vacuum and hydrogen," Tribology Letters 4 (1998) pp. 175-188.

[6] M.B. Petersen and R.L. Johnson, "PbO and Other Metal Oxides as Solid Lubricants for Temperatures to 1000 F," ASME/ASLE paper number 56-LC10 (1956).

[7] F.H. Stott, and G.C. Wood, "The Influence of Oxides on the Friction and Wear of Alloys," Tribology International, 1978, p. 211.

[8] M.N. Gardos, H. Hong and W.O. Winer, "The Effect of Anion Vacancies on the Tribological Properties of Rutile (TiO2-x), Part II: Experimental Evidence,” Tribology Transactions, vol. 36, no. 2, pp. 209-220, April 1990.

[9] M. Woydt, M. Dogigli and P. Agatonovic, "Concepts and Technology Development of Hinge Joints Operated Up to 1600 C in Air," Tribology Transactions, vol. 40, 4, pp. 643-646, October 1997.

[10] E.R. Zaretsky, Tribology for Aerospace Applications, Chapter 3.9 “Cryogenic Ball Bearings,” pp. 128-132, STLE, Park Ridge, IL 1997.

[11] DOD contract number WL F33615-85-C-5087, B.D. McConnell and K.R. Mecklenburg project managers.

[12] S.V. Pepper, "Effect of electronic structure of the diamond surface on the strength of the diamond-metal interface," J. Vac. Science Technology, 20(3), March 1982, pp. 643-646. 


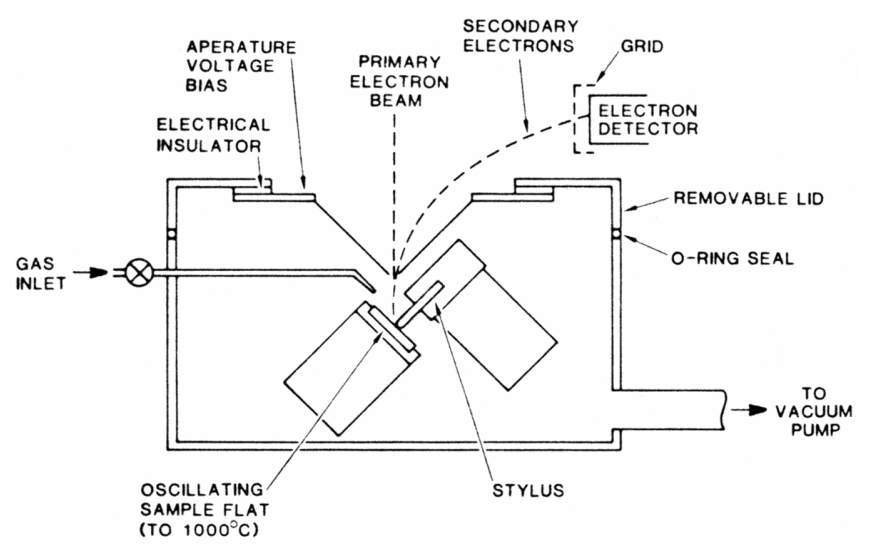

Figure 1. Schematic of SEM Tribotester. From reference 8.
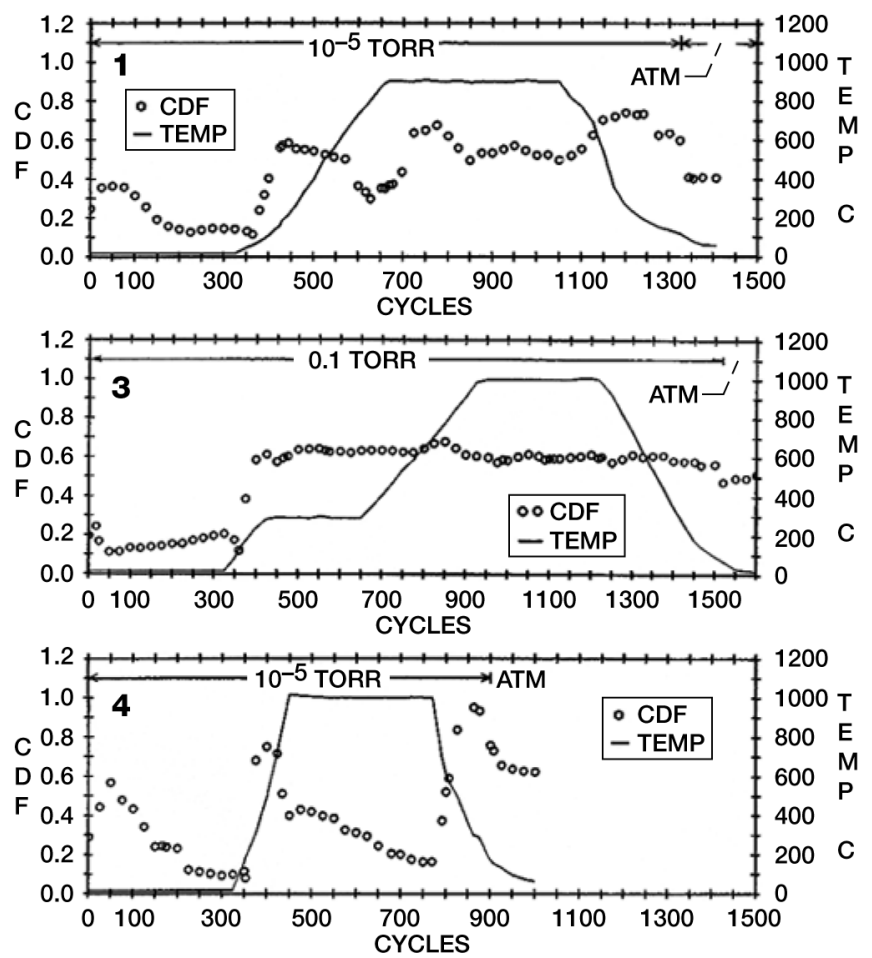

Figure 2. Friction coefficients from model experiments for Rutile under varying temperature and atmospheric conditions adapted from reference 8. 


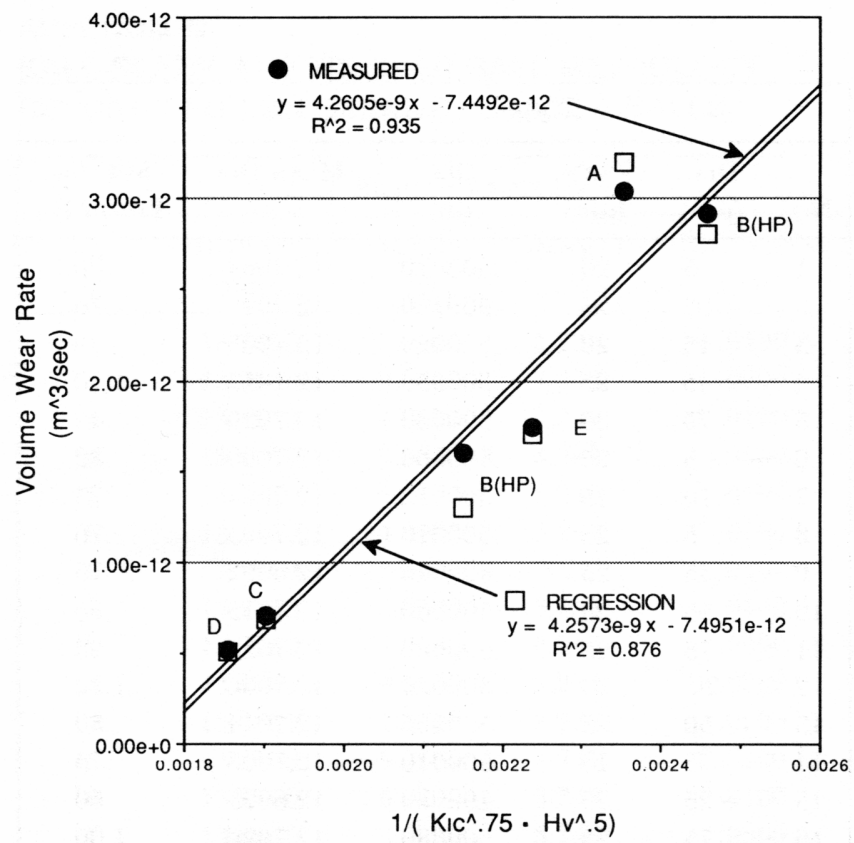

Figure 3. Relationship between silicon nitride volumetric wear and the Evans-Wilshaw wear relationship. Adapted from reference 4. 


\section{1 and 0.3 Torr $\mathrm{PH}_{2}$ (fourth round)}
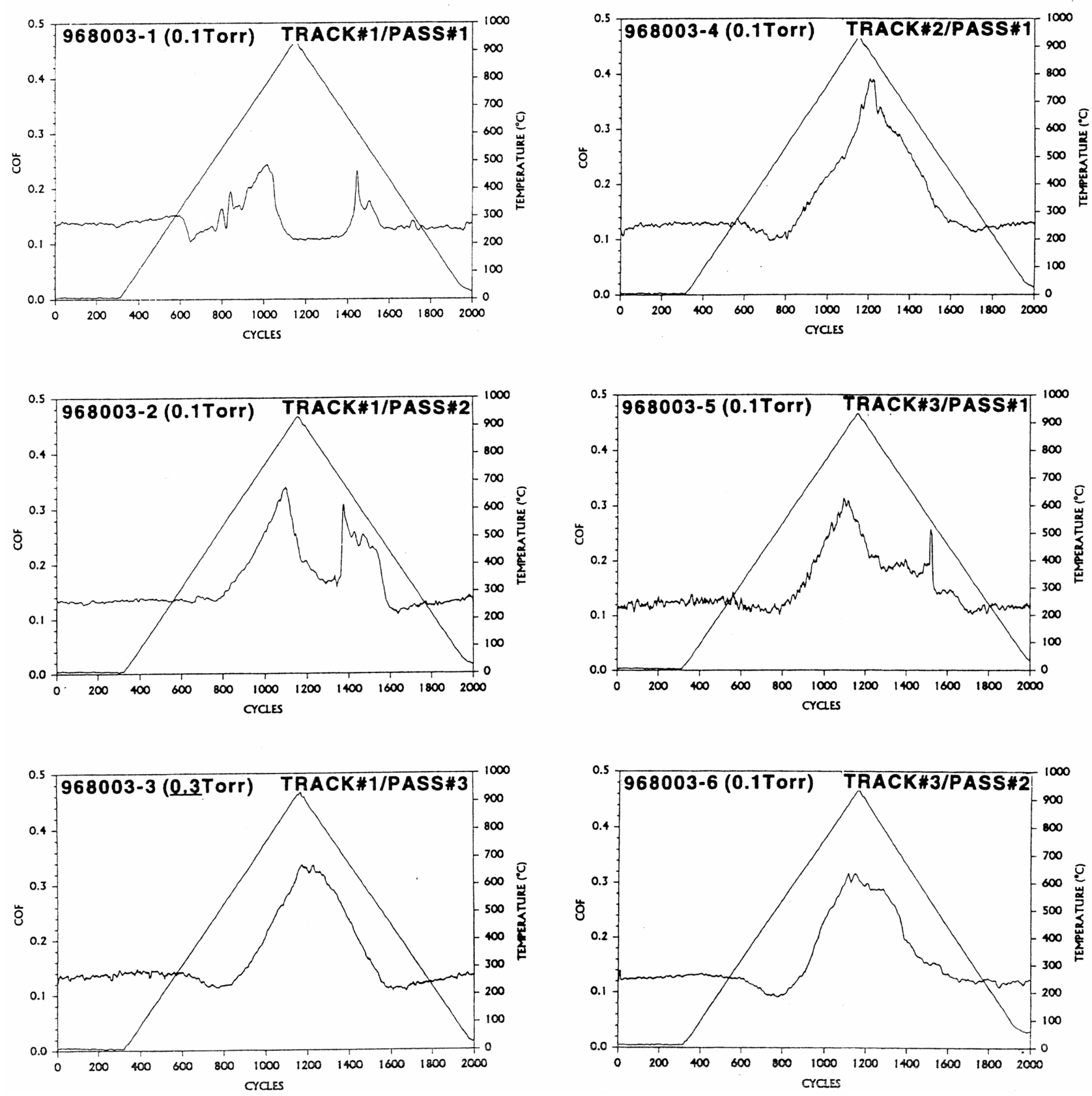

Figure 4. Friction of diamond at varying temperatures and partial pressures of hydrogen. Adapted from reference 5. 